\title{
DYNAMO GENERATION OF GALACTIC MAGNETIC FIELDS
}

\author{
KARL JOHAN DONNER \\ Observatory and Astrophysics Laboratory, University of Helsinki \\ Tähtitorninmäki, SF-00130 Helsinki, Finland
}

\begin{abstract}
I give some results of calculations of the magnetic fields generated by dynamo processes in galaxy models consisting of a turbulent disc embedded in a low-conductivity halo. The field configuration is found to be strongly dependent on the turbulent structure of the disc.
\end{abstract}

Some results of a study of dynamo processes in realistic galaxy models are presented. The ultimate goal of this investigation is to get a better understanding of the origin and maintenance of galactic magnetic fields. However, my concern here is not so much to determine precise conditions for the excitation of various modes, but rather to get a general view of what kind of field structures are expected on the basis of simple kinematic dynamo models for galaxies. In particular, if galactic magnetic fields are generated by dynamo processes, then observed fields should correspond to the dominant (fastest growing or most slowly decaying) mode of the dynamo equation.

The basic model of a galaxy is described by a distribution of turbulent magnetic diffusivity, $\alpha$ effect, and rotational velocity. The turbulent diffusivity is written as the sum of a spherical and a disc-like part. The $\alpha$ effect is assumed to have the same scale height and radial distribution as the disc diffusivity. I use two different models for the disc: in the full disc models the gas density remains constant at the centre, whereas in the ring models the disc has an inner edge and the centre is empty of gas. The rotation curve is a Brandt curve constant on cylinders.

For details on the computational method, see Donner \& Brandenburg (1989) and references therein.

\section{Results}

- In the full disc models the dominant modes are of A0 symmetry. The field is essentially concentrated within one disc thickness of the centre. The structure of the modes is found to be almost independent of the rotation curve, which indicates that they are excited by the $\alpha^{2}$ mechanism.

- In the ring models the dominant mode (for positive $\alpha$ ) is of type S0, followed by S1. These modes are excited by a combination of the $\alpha^{2}$ and the $\alpha \omega$ mechanisms. 
- There appears to be a general tendency for the dominant modes to become localised near a radius of maximum excitation (measured by $\alpha^{2}$ or $\alpha \omega$ ).

- In no case have bisymmetric modes been found to be dominant. However, if they are somehow generated (e.g. in galaxy mergers), they may survive for an appreciable time.
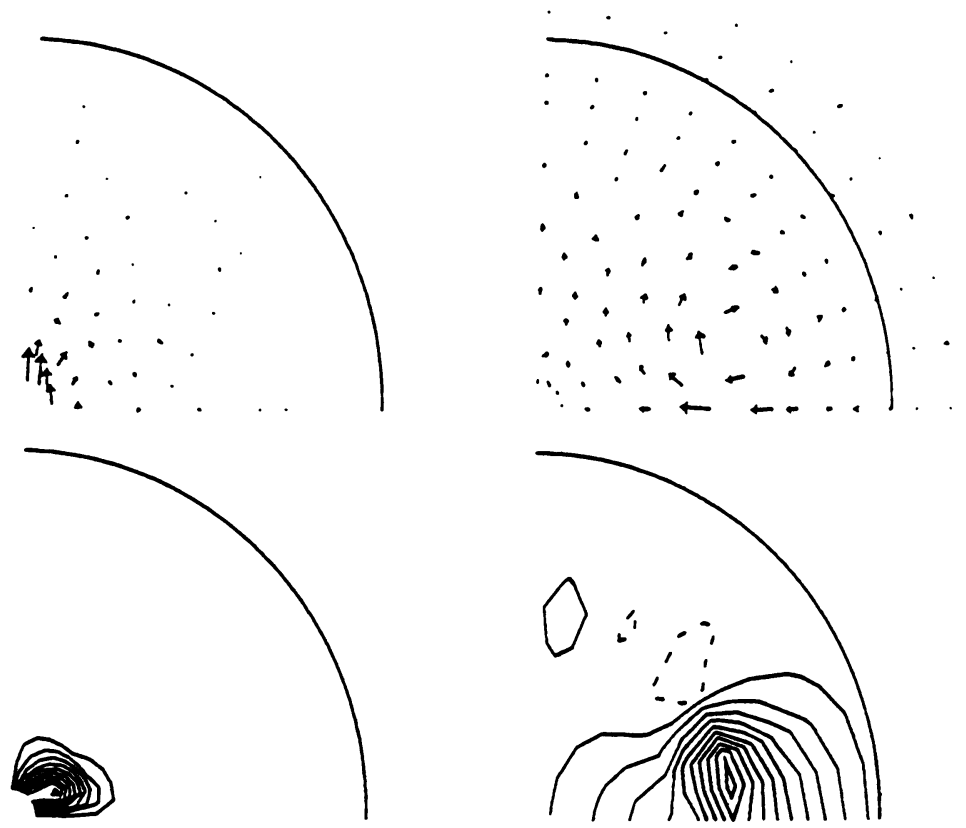

Figure 1: Magnetic field configuration of dominant modes in the full disc (left) and ring models (right). The upper panel shows field vectors of the meridional field, the lower panel shows contours of constant azimuthal field.

\section{Reference}

Donner, K.J. and Brandenburg, A. (1989) 'Magnetic Field Structure in Differentially Rotating Discs', Geophys. Astrophys. Fluid Dyn., (in press). 